\title{
COMPARACIÓN ANATÓMICA E HISTOQUÍMICA DE LA MADERA DEL GUAYACÁN MEDICINAL PROVENIENTE DE MUESTRAS DE MERCADOS CON LA DE GUAIACUM COULTERI A. GRAY
}

\author{
Lucía Yoscelina Centeno-Betanzos ${ }^{1,3}$, Alicia Enriqueta \\ Brechú-Franco ${ }^{1}$, Helia Reyna Osuna-Fernández ${ }^{1}$, \\ Guillermo Laguna-Hernández ${ }^{1}$, Calixto León-Gómez ${ }^{2}$

\begin{abstract}
${ }^{1}$ Universidad Nacional Autónoma de México, Facultad de Ciencias, Departamento de Ecología y Recursos Naturales, 04510 México, D.F., México. ${ }^{2}$ Universidad Nacional Autónoma de México, Instituto de Biología, Departamento de Botánica, Apdo. postal 70-233, 04510 México, D.F., México.

${ }^{3}$ Autor para la correspondencia: yosfc@yahoo.com.mx
\end{abstract}

\section{RESUMEN}

El guayacán como planta medicinal se recomienda para problemas de salud derivados de la diabetes mellitus. En la literatura, la especie que cuenta con más reportes con el nombre común de guayacán es Guaiacum coulteri. Se caracterizaron anatómica e histoquímicamente muestras de la madera de guayacán procedentes de cinco mercados públicos de la Ciudad de México y una de G. coulteri obtenida de la xiloteca MEXUw, del Instituto de Biología de la Universidad Nacional Autónoma de México. Al contrastar la estructura anatómica de los especímenes comprados con los de la xiloteca, ninguna correspondió con G. coulteri y además entre ellos fueron diferentes. En la comparación histoquímica se encontró mayor contenido lipídico y glucoproteíco en las células de parénquima de las muestras de mercado que en el escaso tejido de G. coulteri. Con este trabajo se demostró la importancia de caracterizar las especies que se comercializan como medicinales mediante su análisis anatómico, para una plena identificación. La reacción histoquímica realizada permitió resaltar con mayor precisión sus diferencias estructurales y dar información acerca de su estructura celular; sin embargo, estos datos podrían complementarse con un estudio fitoquímico posterior que provea mayor información acerca de los compuestos de cada muestra.

Palabras clave: anatomía de la madera, Guaiacum coulteri, histoquímica, plantas medicinales. 


\begin{abstract}
The medicinal plant guayacan is recommended for health problems derived from diabetes mellitus. The species most often associated in the literature with the common name guayacan is Guaiacum coulteri. For this study, anatomical and histochemical comparisons were made of wood samples from five markets in Mexico City and of Guaiacum coulteri wood deposited in the xylarium MEXUw, housed at the Instituto de Biología, Universidad Nacional Autónoma de México. The market samples were anatomically dissimilar, not only to Guaiacum coulteri but also to each other. The histochemical comparison showed higher lipid and glucoprotein contents in the parenchyma of the market samples than in $G$. coulteri, whose parenchyma is scarce. These results demonstrate the importance of anatomical characterization of species that are commercialized for medicinal use in order to assure an accurate identification. The histochemical reactions of samples enabled the structural differences among them to be observed with greater precision, and provided more information on their cellular composition; however, these results could be complemented with a subsequent phytochemical study that would provide more information on the compounds in each sample.
\end{abstract}

Key words: Guaiacum coulteri, histochemistry, medicinal plants, wood anatomy.

\title{
INTRODUCCIÓN
}

Ramawat et al. (2004) señalan que la Organización Mundial de la Salud (OMS) estima que $80 \%$ de la población mundial confía en la medicina tradicional y en fármacos basados en compuestos vegetales para resolver sus principales necesidades de salud. En México se calcula que $60 \%$ de la gente utiliza la herbolaria medicinal. En zonas urbanas de la República Mexicana la forma común de conseguir plantas medicinales es acudiendo a los mercados públicos, en los que siempre hay uno o más locales comerciales donde pueden adquirirse. En la Ciudad de México existen alrededor de 312 mercados públicos que cubren la mayor parte del abasto de ese recurso para los capitalinos. En algunos casos, esta forma popular de compra puede ser riesgosa para el consumidor, ya que en el comercio existen diferentes especies botánicas conocidas con el mismo nombre común. Algunas de ellas, principalmente las que se venden secas o troceadas, presentan una similitud morfológica que no permite distinguirlas. Esto facilita que un recurso pueda ser substituido por otro cuando uno escasea, sin que el comprador sea informado o lo note. Es por ello que al comprar 
una planta o mezclas secas de ellas para remedio de una afección o enfermedad, se corra el peligro de no obtener el efecto requerido o incluso tener uno antagónico y poner en riesgo la salud de los consumidores (Carmona-Jiménez, 1992).

Un ejemplo de lo anterior se observa en el comercio de la madera y/o corteza del guayacán o guayacán amarillo, que se recomienda para tratar problemas relacionados con la diabetes mellitus. Ésta se expende sola o en mezclas con otras plantas, lo que dificulta su reconocimiento, ya que al estar troceadas, se pierden características con las que pudieran ser reconocidas. En la literatura, el nombre de guayacán se asigna a más de 20 especies botánicas diferentes pero principalmente a Guaiacum coulteri de la familia Zygophyllaceae (Centeno-Betanzos, 2007), la cual cuenta con estudios farmacológicos que muestran un efecto hipoglucemiante (Ramos et al., 1992). En varios trabajos se ha demostrado que la introducción de nuevos elementos vegetales en el comercio es muy frecuente (Anaya, 1991; García, 1992; Manzanero, 1995; Gutiérrez y Betancourt, 2002) y que los estudios anatómicos son de gran ayuda para conocer la identidad de la planta, especialmente cuando no se cuenta con flores o frutos, siendo útil para detectar la adulteración, fraude o sustitución de un producto (Carmona-Jiménez, 1992). Es por lo anterior que se decidió caracterizar la anatomía e histoquímica de la madera que se vende como guayacán o guayacán amarillo en mercados importantes de la Ciudad de México y compararla con la de G. coulteri para validar su correspondencia.

\section{MATERIALES Y MÉTODOS}

Trabajo de campo

Se visitaron cinco mercados de la Ciudad de México en donde se venden plantas medicinales: Sonora, Jamaica, Xochimilco, Pasaje Catedral y M. Múzquiz (San Ángel) y se compraron muestras de guayacán o guayacán amarillo en tres locales elegidos al azar de cada uno de ellos. Por otra parte, la madera de G. coulteri se obtuvo de la xiloteca MEXUw del Instituto de Biología de la Universidad Nacional Autónoma de México (UNAM).

\section{Microtecnia}

Todas las muestras se seccionaron en trozos de $1 \mathrm{~cm}^{2}$ y se ablandaron en agua hirviendo por 60 min y posteriormente en etilendiamina a 10\% hirviendo por 60 a 90 min (Carlquist, 1982 y Kukachka, 1977). La inclusión se hizo en polietilenglicol (PEG) gradual (León-Gómez, 1997). Se obtuvieron cortes de 15 a $20 \mu \mathrm{m}$ de grosor 
en tres planos de orientación con un microtomo de deslizamiento. Para la descripción anatómica se usó la tinción con safranina acuosa a 1\%. Las laminillas se montaron con resina sintética Entellan. Para el disociado de la madera se seccionaron astillas delgadas de cada muestra, las cuales se pusieron en solución de Jeffrey (trióxido de cromo y ácido nítrico 1:1 a 10\% en solución acuosa) por 24 y $48 \mathrm{hrs}$, dependiendo de la dureza. Posteriormente se montaron en resina sintética.

Para la descripción cualitativa externa de la madera, se siguieron los criterios usados por Barajas-Morales y León-Gómez (1989). Los promedios obtenidos para cada una de las características se calcularon con 25 mediciones hechas en un microscopio óptico marca Zeiss. La terminología, estándares y algunos aspectos de la organización celular, se basaron en las propuestas por el Comité de Nomenclatura de la Asociación Internacional de Anatomistas de la Madera (Anónimo, 1989).

\section{Histoquímica}

Se usó el azul negro de naftol en solución alcohólica $0.5 \%$ (ANN) para la detección de proteínas, la doble tinción azul negro de naftol y ácido peryódico reactivo de Schiff (ANN-Sch) para reconocer glucoproteínas y polisacáridos insolubles (López et al., 2005), y la tinción cuádruple de Johansen (CJ) para identificar lignina, celulosa, almidón, proteínas y lípidos insolubles (Johansen, 1940).

\section{RESULTADOS}

\section{Anatomía}

A partir de la descripción anatómica de las muestras adquiridas en los mercados, se encontraron marcadas diferencias y similitudes en su estructura celular, principalmente en el arreglo del parénquima axial, número de células del parénquima estratificado y número de estratos por milímetro, lo que permitió clasificarlas en tres grupos:

Grupo 1. En este grupo se incluyen las muestras de los mercados Sonora y Pasaje Catedral.

Descripción cualitativa externa: Color amarillo claro, textura lisa, aromático y ligeramente amargo.

Caracteres microscópicos: (Láminas 1 y 2. Figs. 1, 5, 9 y 13).

Vasos: Porosidad difusa, con poros de contorno ovalado y circular. Éstos se encontraron principalmente en múltiples radiales de 2 y 3 y algunos solitarios (Fig. 
5); fueron escasos, con 3 poros $/ \mathrm{mm}^{2}$ y medianos con un diámetro tangencial de 116 $\mu \mathrm{m}$ en promedio $(86-160 \mu \mathrm{m})$. Elementos de vaso cortos, con longitud promedio de $221 \mu \mathrm{m}(148-309 \mu \mathrm{m})$. Placa de perforación simple con inclinación de 30 a 45․ Punteaduras intervasculares y de vaso a radio alternas y pequeñas, con $6 \mu \mathrm{m}$ de diámetro.

Parénquima axial: Paratraqueal en abundantes bandas confluentes y vasicéntrico de una hilera de células (Fig. 5). Cada banda tuvo 6 estratos celulares en promedio, vistos en corte transversal y con 4 células por serie parenquimatosa (Fig. 1). Cada célula presentó varios cristales poliédricos en cámara.

Parénquima radial: Los radios fueron muy numerosos, $14 / \mathrm{mm}$ en promedio, heterocelulares tipo III, principalmente biseriados y en menor cantidad triseriados (Fig. 1); formados por células procumbentes en el cuerpo y con una hilera de células erectas en ambos márgenes; fueron extremadamente bajos, con una altura promedio de $154 \mu \mathrm{m}(123-205 \mu \mathrm{m})$.

Fibras: Libriformes, moderadamente cortas, con longitud promedio de 811 $\mu \mathrm{m}(494-1087 \mu \mathrm{m})$. Paredes gruesas con $3 \mu \mathrm{m}$ de espesor y $9 \mu \mathrm{m}$ de diámetro tangencial.

Otras características: Se presentó estratificación del parénquima axial, elementos de vaso y radios, con 6 líneas/mm. (Fig. 1).

Grupo 2. En este grupo se incluyen las muestras de los mercados Jamaica y Xochimilco.

Descripción cualitativa externa: color amarillo claro, textura lisa, ligeramente aromático y ligeramente amargo.

Caracteres microscópicos: (Láminas 1 y 2. Figs. 2, 6, 10 y 14).

Vasos: Porosidad semianular a difusa, con poros circulares y ovalados; principalmente solitarios, algunos múltiples radiales, otros en racimos de 2 o 3 células y escasos de 4 o 5 células (Fig. 6); fueron moderadamente numerosos, con 12 poros/ $\mathrm{mm}^{2}$ y medianos con un diámetro tangencial de $105 \mu \mathrm{m}$ en promedio $(74-136 \mu \mathrm{m})$. Los elementos de vaso fueron muy cortos, con longitud promedio de $175 \mu \mathrm{m}$ (111$247 \mu \mathrm{m})$. Placa de perforación simple, con inclinación de 15 a $45^{\circ}$. Punteaduras intervasculares y de vaso a radio alternas y pequeñas, con $6 \mu \mathrm{m}$ de diámetro.

Parénquima axial: Paratraqueal en bandas confluentes (Fig. 6). Cada banda con 6 a 8 estratos celulares de amplitud en promedio, vistos en corte transversal y principalmente con 2 células por serie parenquimatosa (Fig. 2). Ocasionalmente con células fusiformes. Cada célula presentó varios cristales poliédricos en cámara.

Parénquima radial: Los radios fueron numerosos, $12 / \mathrm{mm}$ en promedio, homocelulares, principalmente biseriados y escasamente triseriados (Fig. 3); formados 
por células procumbentes; extremadamente bajos, con una altura promedio de 137 $\mu \mathrm{m}(98-173 \mu \mathrm{m})$.

Fibras: Libriformes, moderadamente cortas, con longitud promedio de 876 $\mu \mathrm{m}(703-1235 \mu \mathrm{m})$. Paredes gruesas, con $3 \mu \mathrm{m}$ de espesor y $8 \mu \mathrm{m}$ de diámetro tangencial.

Otras características: Se presentó estratificación del parénquima axial, elementos de vaso y radios, con 8 líneas/mm. (Fig. 2).

Grupo 3. Este grupo incluye una sola muestra del mercado Jamaica.

Descripción cualitativa externa: Color amarillo claro, textura lisa, sin olor, sin sabor.

Caracteres microscópicos: (Láminas 1 y 2. Figs. 3, 7, 11 y 15).

Vasos: Porosidad difusa, con poros ovalados; se observaron principalmente solitarios, más escasos los múltiples radiales de 2, 3 y hasta 4 células (Fig. 7); moderadamente numerosos, con 9 poros $/ \mathrm{mm}^{2}$ y pequeños con un diámetro tangencial de $70 \mu \mathrm{m}$ en promedio $(62-86 \mu \mathrm{m})$. Los elementos de vaso fueron cortos, con longitud promedio de $227 \mu \mathrm{m}(148-309 \mu \mathrm{m})$. Placa de perforación simple con inclinación de $45^{\circ}$. Punteaduras intervasculares y de vaso a radio alternas y pequeñas con 4 a $5 \mu \mathrm{m}$ de diámetro.

Parénquima axial: Paratraqueal de distribución aliforme y marginal (Fig. 7). Cada banda con 3 a 6 estratos celulares de amplitud en promedio, vistos en corte transversal y con series de 2 a 4 células por serie parenquimatosa (Fig. 3). Cada célula presentó de 4 a 6 cristales poliédricos en cámara. Los cristales en este grupo fueron de gran tamaño, de $15 \mu \mathrm{m}$ en promedio (13-18 $\mu \mathrm{m})$, medidos en corte tangencial a $40 x$.

Parénquima radial: Los radios fueron abundantes, 17/mm, homocelulares, principalmente triseriados y tetraseriados y en menor cantidad multiseriados (Fig. 3); se observaron formados por células procumbentes; extremadamente bajos, con una altura promedio de $385 \mu \mathrm{m}(253-495 \mu \mathrm{m})$.

Fibras: Fibrotraqueidas, largas con longitud promedio de $774 \mu \mathrm{m}$ (518-988 $\mu \mathrm{m})$. Paredes gruesas, de $3 \mu \mathrm{m}$ de espesor y $11 \mu \mathrm{m}$ de diámetro tangencial en promedio. Presentaron abundantes y pequeñas punteaduras areoladas en corte tangencial (3 a $5 \mu \mathrm{m})$.

Otras características: No hubo estratificación (Fig. 3). Se observaron anillos de crecimiento debido al parénquima marginal y canales intercelulares de origen traumático con goma, alineados en bandas tangenciales en algunas zonas del xilema. 

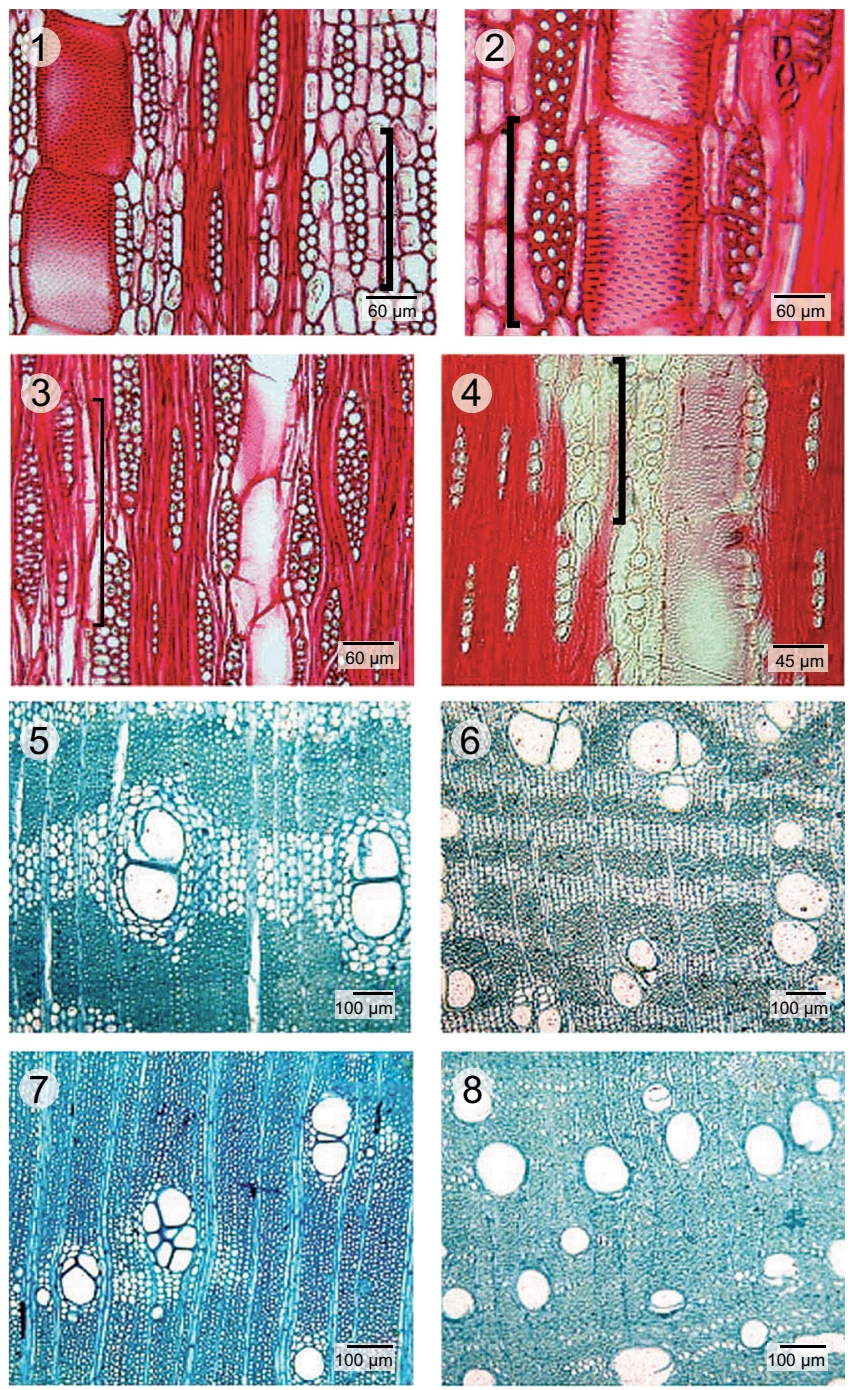

Lámina 1. Anatomía e histoquímica de la madera del guayacán de mercado y Guaiacum coulteri. Maderas teñidas con safranina (Figs. 1-4) y con azul negro de naftol, que tiñe proteínas de azul (Figs. 5-8). Grupo 1: Fig. 1. Parénquima paratraqueal con 4 células por serie parenquimatosa (corchete); Fig. 5. Poros circulares en agregados de 2 y 3. Grupo 2: Fig. 2. Parénquima paratraqueal con 2 células por serie parenquimatosa (corchete); Fig. 6. Poros circulares dispuestos en agregados y algunos solitarios. Grupo 3: Fig. 3. Parénquima paratraqueal con series de 2 a 4 células por serie parenquimatosa (corchete); Fig. 7. Poros ovalados en agregados. G. coulteri: Fig. 4. Parénquima paratraqueal con 2 células por serie parenquimatosa (corchete); Fig. 8. Poros ovalados y solitarios. 
Guaiacum coulteri: Muestra de G. coulteri obtenida de la xiloteca MEXUw del Instituto de Biología de la Universidad Nacional Autónoma de México.

Descripción cualitativa externa: Color amarillo, textura lisa, sin olor y de sabor ligeramente amargo. Veteado irregular, contraste entre albura de color amarillo y duramen de color pardo verdoso.

Caracteres microscópicos: (Láminas 1 y 2. Figs. 4, 8, 12 y 16).

Vasos: Porosidad difusa, con poros ovalados y solitarios (Fig. 8), numerosos, con 35 poros $/ \mathrm{mm}^{2}$ y pequeños con un diámetro tangencial de $98 \mu \mathrm{m}$ en promedio (74-123 $\mu \mathrm{m})$. Los elementos de vaso fueron cortos con longitud promedio de $140 \mu \mathrm{m}$ $(86-185 \mu \mathrm{m})$. Placa de perforación simple con una inclinación de $15^{\circ}$. Punteaduras intervasculares y de vaso a radio alternas, areoladas y pequeñas con 4 a $6 \mu \mathrm{m}$ de diámetro.

Parénquima axial: Paratraqueal, escaso, aliforme y confluente con 1 o 2 estratos celulares, vistos en corte transversal (Fig. 8) y con 2 células por serie parenquimatosa (Fig. 4). Cada célula presentó un cristal estiloideo.

Parénquima radial: Los radios fueron muy abundantes, $26 / \mathrm{mm}$ en promedio, homocelulares y exclusivamente uniseriados (Fig. 4), formados por células procumbentes y extremadamente bajos con una altura promedio de $95 \mu \mathrm{m}(77-123 \mu \mathrm{m})$.

Fibras: Fibrotraqueidas largas, con longitud promedio de $811 \mu \mathrm{m}(642-1050$ $\mu \mathrm{m})$. Paredes gruesas con $3 \mu \mathrm{m}$ de espesor y $9 \mu \mathrm{m}$ de diámetro tangencial. Presentaron abundantes y pequeñas punteaduras ( 3 a $5 \mu \mathrm{m}$ ) areoladas en corte tangencial.

Otras características: Hubo estratificación del parénquima axial, elementos de vaso y radios, con 13 líneas/mm (Fig. 4). Madera muy dura, fuerte y durable.

\section{Histoquímica}

El azul negro de naftol (ANN) es empleado para la identificación de proteínas. (Lámina 1. Figs. 5 y 8). En todas las muestras se observó reacción intensa en paredes y citoplasma del parénquima axial y radial. En los grupos 1 y 2 las bandas confluentes se definieron con claridad del parénquima que se intercala con las fibras (Figs. 5 y 6). En G. coulteri las fibras se tiñeron fuertemente, lo que produjo un color homogéneo en todo el tejido por su abundancia, resaltando la forma de los vasos y el escaso parénquima paratraqueal de tipo aliforme a su lado (Fig. 8). Sobresalió la diferencia en la presencia de proteínas celulares entre los grupos y G. coulteri.

El Schiff-azul negro de naftol (Sch-ANN) es usado para la identificación de glucoproteínas y polisacáridos insolubles (Lámina 2. Figs. 9 y 12). En todas las muestras, excepto en G. coulteri, a pesar de que el citoplasma del parénquima radial y axial reaccionó con ambos colorantes, dominó la tinción con Schiff, lo que indicó 

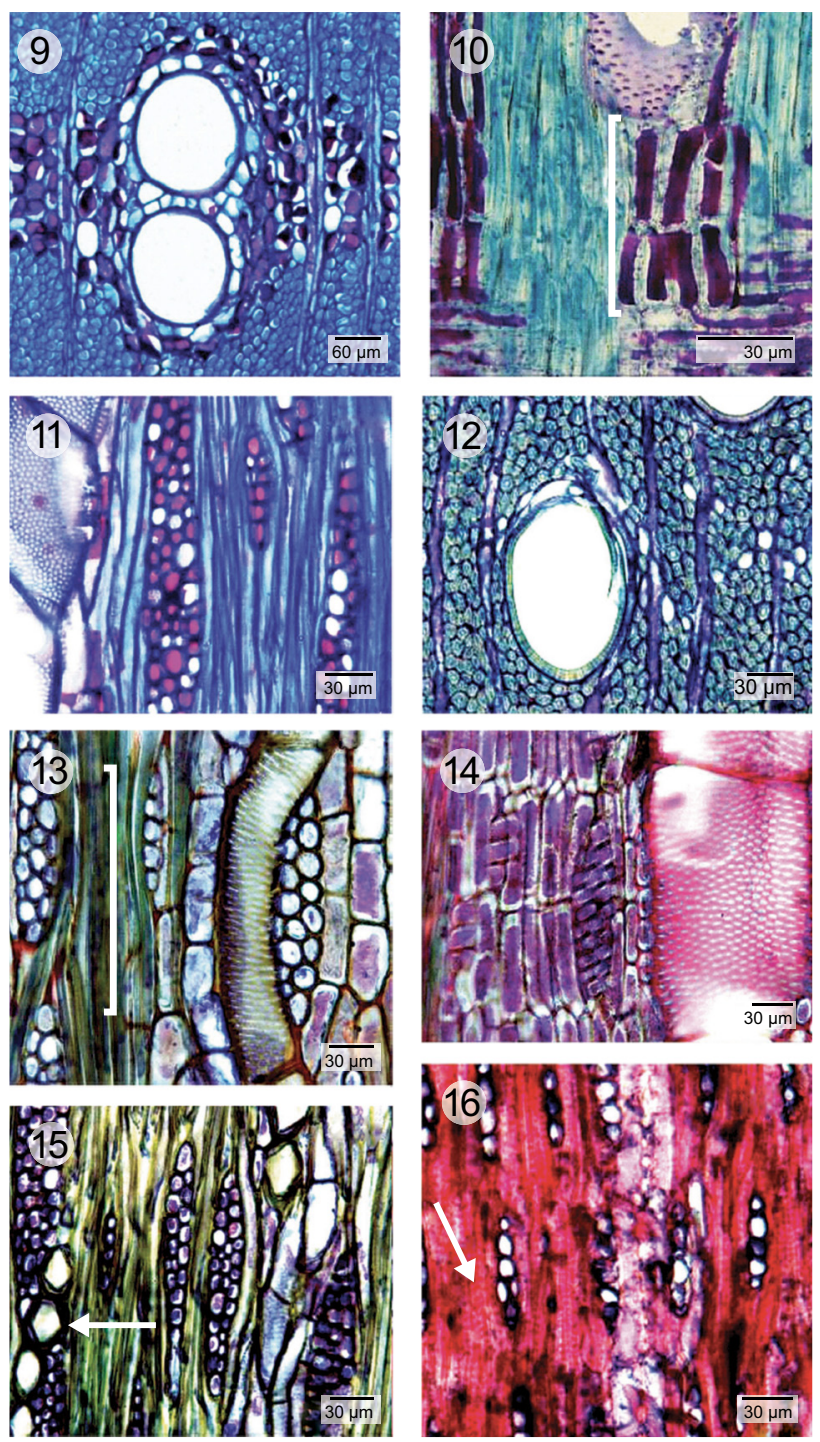

Lámina 2. Histoquímica de la madera del guayacán de mercado y de Guaiacum coulteri. Maderas teñidas con Schiff-azul negro de naftol, que tiñe proteínas de azul y glucoproteínas y polisacáridos en rosa mexicano (Figs. 9-12); en todas las muestras el parénquima axial y radial reacciona a ambos colorantes y las fibras solo al azul. Muestras teñidas con la cuádruple de Johansen, que colora de verde a la celulosa, de morado al contenido glucoprotéico (Figs. 13-16). Grupo 1: Figs. 9 y 13. Fibras gelatinosas (corchete). Grupo 2: Figs. 10 y 14. Parénquima con contenido glucoprotéico (corchete). Grupo 3: Figs. 11 y 15. Cristales (flecha) y fibrotraqueidas celulósicas. G. coulteri: Figs. 12 y 16. Fibrotraqueidas lignificadas (flecha). 
el predominio de carbohidratos celulares, que al contrario de las fibras, resaltó la reacción al azul negro de naftol debido a la abundancia de proteínas presentes en estas células. (Figs. 9, 10 y 11).

La reacción de ambos colorantes en la pared de los vasos hizo más visible las punteaduras (Fig. 10). En el escaso parénquima de G. coulteri, hubo dominancia del azul, lo que fue característico para esta muestra (Fig. 12). El citoplasma del parénquima paratraqueal del grupo 3 presentó un contenido en forma de mucílago.

La tinción cuádruple de Johansen permitió distinguir celulosa, lignina, proteínas y almidón (Lámina 2. Figs. 13 y 16). En los grupos 1 y 2 los contenidos citoplásmicos del parénquima axial y radial se tiñeron de color rosa a morado en diferente intensidad, debido al contenido celular de tipo glucoproteico. Las fibras, principalmente de los grupos 1 y 3, lo hicieron fuertemente de verde, lo que destacó su composición celulósica (Figs. 13, 14 y 15). En G. coulteri, las fibrotraqueidas se tiñeron totalmente de color rojo debido a la abundancia de lignina (Fig. 16). En el citoplasma del parénquima axial del grupo 1 se lograron distinguir cuerpos esferoidales similares a amiloplastos, coloreados en violeta en diferentes intensidades (Fig. 13). El citoplasma del parénquima radial se tiñó de morado debido a la presencia de glucoproteinas (Figs. 13 y 16). En corte tangencial, en los extremos del parénquima radial sobre la célula erecta del grupo 1 se observó un material teñido de rojo, que indicó la presencia de lignina (Fig. 11). En el grupo 3 destacaron los cristales en las células de parénquima (Fig. 15). En G. coulteri tanto la pared de los vasos como las abundantes fibrotraqueidas se tiñeron de rojo por la presencia de lignina, lo que homogenizó la coloración rojiza de todo el tejido. El escaso citoplasma de las células de los radios y del parénquima axial se colorearon de violeta, por el alto contenido de proteínas (Fig. 16).

\section{DISCUSIÓN}

Comparación anatómica entre las muestras del mercado y la madera de Guaiacum coulteri

Aunque las muestras de mercado presentaron diferencias morfológicas cualitativas, éstas no fueron suficientemente contundentes para permitir la distinción de los diferentes grupos con facilidad o a simple vista. Esto confirma el hecho de que al momento de comprar guayacán en los mercados, es muy probable que el consumidor no logre notar la diferencia entre la madera de una y otra especie. Sin embargo, por medio de los caracteres microscópicos se encontraron diferencias importantes en 
todos los elementos celulares que constituyen la madera, lo que permitió clasificar las muestras de los mercados en tres grupos. Sobresalió el hecho de que hubo mayor cantidad de parénquima paratraqueal en las maderas de los grupos 1 y 2 que en el 3 y en G. coulteri, pero estos últimos presentaron más tejido de esclerénquima. Además, G. coulteri superó a todas en abundancia de fibrotraqueidas y cristales que le confieren su dureza.

A pesar de que ninguna estructura celular de las maderas de guayacán compradas en los mercados coincidió con la de G. coulteri, fueron tan características que, por comparación anatómica con especies reportadas por Carmona-Jiménez (1997) y laminillas de la colección de la xiloteca MEXUw del Instituto de Biología de la UNAM, fue posible encontrar que el arreglo celular de la madera del grupo 3 coincidía con el del género Citrus de la familia Rutaceae. Esto hace probable que la muestra sea de Citrus limetta, debido a que hay reportes que indican que la madera de esta especie se comercializa en estos mercados y también se recomienda contra la diabetes, aunque no se le conoce como guayacán (Carmona-Jiménez, 1992; Aguilar y Xolalpa, 2002; Andrade-Cetto y Heinrich, 2005). De la misma forma, por comparación anatómica de los grupos 1 y 2 con las laminillas existentes en la colección de la xiloteca y las reportadas por Barajas-Morales y León-Gómez (1989), se encontró que su estructura celular coincide con la de las especies del género Lonchocarpus (Fabaceae). Así, es posible que las maderas incluidas en el grupo 2 sean de Lonchocarpus cruentus, lo cual se apoya en los registros de Aguilar y Xolalpa (2002) y Andrade-Cetto y Heinrich (2005), quienes reportan a Lonchocarpus cruentus como guayacán y con uso medicinal contra la diabetes. Puesto que los grupos 1 y 2 corresponden a especies del género Lonchocarpus, sería recomendable llevar a cabo el estudio fitoquímico y farmacológico de estas plantas, para evaluar su acción en el control de la diabetes como se reporta en los estudios etnobotánicos.

No se realizaron análisis estadísticos más amplios, debido a que la descripción anatómica permitió establecer las diferencias entre las muestras.

\section{Comparación histoquímica}

En la comparación histoquímica de los tres grupos del mercado y G. coulteri se observó que las respuestas celulares resaltaron aún más las diferencias estructurales. En G. coulteri, la fuerte reacción del azul negro de naftol en las abundantes fibrotraqueidas lignificadas se debió al elevado contenido proteico en sus paredes, y el escaso parénquima axial se coloreó débilmente con el reactivo doble, Schiff-azul negro de naftol y con la tinción cuádruple de Johansen, en comparación con las demás muestras. En todos los casos hubo reacción positiva de proteínas y carbohi- 
dratos en el parénquima axial y radial, lo que señaló que el contenido celular es de composición glucoproteica. El material ergástico en todas las muestras se observó mejor por la ausencia de coloración. Las diferencias en la cantidad de células de parénquima axial y radial, y por lo tanto en la proporción de sus compuestos entre G. coulteri y las demás maderas, fueron más visibles con el uso de las técnicas histoquímicas. De esta manera, se pudo poner en evidencia que las sustancias con posible actividad medicinal como las glucoproteínas existentes en el parénquima, fueron mucho más abundantes en la estructura celular de las maderas del mercado que en las de G. coulteri.

Con la tinción cuádruple de Johansen se conoció y diferenció la composición de los contenidos celulares, ya que mientras que las fibrotraqueidas de G. coulteri se tiñeron de rojo por la presencia de lignina, las fibras de muestras de mercado se colorearon principalmente de verde por la existencia de celulosa. A este tipo de fibras que en las paredes conservan celulosa, se les conoce como fibras gelatinosas, las cuales son parte de la madera de reacción presente en las ramas. Lo anterior confirma que la madera con ese tipo de fibras provenía de ramas como se describió en la morfología.

La aplicación por separado de la tinción azul negro de naftol (ANN) para detectar la presencia de proteínas y la doble reacción Schiff-azul negro de naftol (SchANN) para distinguir glucoproteínas, así como el uso de la cuádruple de Johansen en la madera, reportada por primera vez en este trabajo con excelentes resultados, permitió localizar en los tejidos, contenidos celulares de importancia medicinal (Laguna-Hernández, et al., 1995).

De esta forma, se confirma que la histoquímica es una herramienta de gran ayuda en la identificación de la presencia, ubicación y distribución de posibles compuestos de importancia fitoquímica en los tejidos vegetales, así como para un mejor realce y contraste de las estructuras celulares que facilita la descripción anatómica. Consideramos que la aplicación de tales técnicas en conjunto con las que ya se utilizan puede aportar conocimiento valioso en la caracterización de las partes vegetales utilizadas en la medicina tradicional (Estrada, 1982; Laguna-Hernández et al., 1995), así como en la información que se integra en la farmacognosia de las farmacopeas.

\section{CONCLUSIONES}

La comparación anatómico-histoquímica de las maderas de guayacán obtenidas de mercados con la de Guaiacum coulteri evidenció que las especies que se están comercializando actualmente con este nombre en los principales mercados de 
la Ciudad de México, no corresponden a G. coulteri. Se reconocieron tres grupos estructuralmente distintos que presentaron afinidades anatómicas con otros géneros, de manera que las maderas incluidas en los grupos 1 y 2 corresponden a Lonchocarpus (Fabaceae) y la del grupo 3 a Citrus (Rutaceae).

\section{AGRADECIMIENTOS}

A la M. en C. Josefina Barajas Morales, encargada de la colección de la xiloteca del Instituto de Biología de la Universidad Nacional Autónoma de México, por las muestras de madera de G. coulteri y L. cruentus y por las facilidades en la realización de este trabajo. Al personal del laboratorio de Ficología de la Facultad de Ciencias de la Universidad Nacional Autónoma de México por facilitar el equipo de fotografía y al Dr. David S. Gernandt del Instituto de Biología de la Universidad Nacional Autónoma de México por la revisión del manuscrito.

\section{LITERATURA CITADA}

Aguilar, A. y S. Xolalpa. 2002. La herbolaria mexicana en el tratamiento de la diabetes. Ciencia 53(3): 24-35.

Anaya, M. 1991. Estudio etnobotánico del complejo quina en México. Tesis de licenciatura. Facultad de Ciencias, Universidad Nacional Autónoma de México. México, D.F., México. 164 pp.

Andrade-Cetto, A. y M. Heinrich. 2005. Mexican plants with hypoglycaemic effect used in the treatment of diabetes. J. Ethnopharmacol. 99: 325-348.

Anónimo. 1989. List of microscopic features for hardwood identification. International Association of Wood Anatomists Bull. 10(3): 219-332.

Barajas-Morales, J. y C. León-Gómez. 1989. Anatomía de maderas de México: especies de una selva baja caducifolia. Publicaciones Especiales 1. Universidad Nacional Autónoma de México. México, D.F., México. 128 pp.

Carlquist, S. 1982. The use of ethylenediamine in softening hard plant structures for paraffin sectioning. Stain Technology 54(5): 311-317.

Carmona-Jiménez, M. L. 1992. Estudio anatómico, morfológico y etnobotánico de algunas maderas del mercado Sonora. Tesis de licenciatura. Facultad de Ciencias, Universidad Nacional Autónoma de México. México, D.F., México. 182 pp.

Centeno-Betanzos, L. Y. 2007. Comparación anatómica e histoquímica de corteza y madera de guayacán de mercado de uso medicinal con Guaiacum coulteri (Zygophyllaceae) de la xiloteca MEXUw. Tesis de licenciatura. Facultad de Ciencias, Universidad Nacional Autónoma de México. México, D.F., México. 103 pp. 
Estrada, E. 1982. Manual de técnicas histológicas. AGT Editores. México, D.F., México. $140 \mathrm{pp}$.

García, B. 1992. Estudio etnobotánico de las cortezas medicinales del estado de Morelos y zonas limítrofes con Puebla y Guerrero. Tesis de licenciatura. Universidad Nacional Autónoma de Morelos. Cuernavaca, Morelos, México. 100 pp.

Gutiérrez, M. y Y. Betancourt. 2002. El mercado de plantas medicinales en México: situación actual y perspectivas de desarrollo. Programa Panamericano de Defensa y Desarrollo de la Diversidad Biológica, Cultural y Social. Buenos Aires, Argentina. Consultado el 4 de marzo de 2011. http://www.prodiversitas.bioetica.org/nota65.htm\#

Johansen, D. 1940. Plant microtechnique. McGraw-Hill. New York, USA. 523 pp.

Kukachka, B. 1977. Sectioning refractory wood for anatomical studies. Note-0236. Forest Service Research of the United State Department of Agriculture - University of Wisconsin. Laramie, Wisconsin, USA. 9 pp.

Laguna-Hernández, G., M. C Pérez-Amador y A. García-Argaez. 1995. Comparative histological and histochemical study of the "Jalapa root". Phyton 57(2): 173-177.

León-Gómez, C. 1997. Arquitectura hidráulica de vitáceas (lianas) de Los Tuxtlas, Veracruz. Tesis de maestría. Facultad de Ciencias, Universidad Nacional Autónoma de México. México, D.F., México. 137 pp.

López, M., J. Márquez y G. Murguía. 2005. Técnicas para el estudio del desarrollo en angiospermas. 2a. Ed. Las Prensas de Ciencias. México, D.F., México. 178 pp.

Manzanero, G. 1995. Estudio de algunas raíces medicinales frescas del mercado Sonora, México, D.F. Tesis de licenciatura. Facultad de Ciencias, Universidad Nacional Autónoma de México. México, D.F., México. 173 pp.

Ramawat, K., K. Sonie y M. Sharma. 2004. Therapeutic potential of medicinal plants: an introduction. In: Ramawat, K. G. (ed.). Biotechnology of medicinal plants: vitalizer and therapeutic. Science Publishers. Enfield, New Hampshire, USA. 302 pp.

Ramos, R., F. Alarcón-Aguilar, A. Lara-Lemus y J. Flores-Saenz. 1992. Hypoglycemic effect of plants used in México as antidiabetics. Arch. Med. Res. 23(1): 59-64. 